\title{
ASPERISPORIUM BLACK SPOT IN CARICA PAPAYA: A NEW DISEASE IN SRI LANKA
}

\author{
N.K.B. ADIKARAM and M. WIJEPALA \\ Department of Botany, University of Peradeniya, Peradeniya.
}

(Received: 31 May 1995; accepted: 11 August 1995)

\begin{abstract}
This paper reports for the first time the occurrence of papaya black spot (Asperisporium caricae) in Sri Lanka, a disease previously confined to South and Central Americas and Africa. In early 1992 we noticed an unusual disease in the mature leaves and the fruits of several papaya cultivars in Hantana located about 2 miles from the Kandy town. The disease was diagnosed as black spot caused by A. caricae and confirmed by carrying out Koch's postulates. The disease is now widespread in several locatlities in the Kandy District and affects almost every papaya cultivar. The disease is characterized by small, black coloured; well-isolated spots on the lower surface of leaves of the lower half of the crown, the intensity of the disease was high in the more mature leaves. There were two other fungi, Sclerospora sp. and Verticillium sp., growing on the spots at certain periods of the year, the mycelium of which had no contact with the leaf tissue. Microscopic studies indicated that the two fungiare probably mycoparasitic on $A$. caricae. The disease was severe during wet weather and the diseased plants fully recovered during prolonged dry spells. The disease appears to be of considerable importance as it reduces photosynthesis area of the leaf affecting the vigour, yield etc. and results in premature fruit drop when young fruits are infected.
\end{abstract}

Key words: Asperisporium, black spot Carica papaya.

\section{INTRODUCTION}

Development of black spot, or "rust" in the leaf and the fruits of Carica papaya due to infection of Asperisporium caricae (Speg.) Maubl. has been reported in Florida $^{1}$ and South America. ${ }^{2}$ The fungus was originally restricted to Central and South America, West Indies and the USA. ${ }^{3}$ However, more recent reports indicate that the disease is wide spread in South Africa ${ }^{4}$ and occurs in Tanzania. ${ }^{5}$ The fungus has recently entered Australia and spread from the north to south (Greg Johnson, personal communication).

There are no reports indicating its occurrence in Asia, though papaya is common in most countries of the South and South East Asia. In this paper the occurrence of this disease in the Central Province of Sri Lanka is reported for the first time.

\section{METHODS AND MATERIALS}

This study was carried out on diseased papaya trees in several locations in close proximity to the Kandy town (Hantana, Peradeniya, Gannoruwa etc.) during 1992-1994. The papaya cultivars under study were not known by name but all of them were common and popular local cultivars. Field investigations were conducted regularly on selected papaya trees in the above mentioned areas for the presence, distribution and severity of symptoms. Disease severity was 
recorded as number of spots per leaf area using an index. Rainfall data for the period of investigation were obtained from the Department of Geography, University of Peradeniya.

Diseased leaves and fruits at different development stages were collected and taken to the laboratory for microscopical and cultural studies. Thin $(2 \mu)$ microtome sections were cut through the diseased areas of leaves at different development stages and stained in hematoxylin for detailed microscopic examination. Attempts were made to isolate the fungi associated with the disease on Potato dextrose Agar, Cook's No. 2 and Czapek Dox Agar medium following surface sterilization with $\mathrm{NaOCl}$ (Chlorex).

For inoculation studies mycelia carefully scraped from the spots with, a) only A. caricae infections, b) A. caricae together with Sclerospora sp. and Verticillium sp., were suspended separately in sterile distilled water. The suspensions were filtered through glass wool and the filtrates were centrifuged twice at $3000 \mathrm{rpm}$ for 3 minutes, each time resuspending the residue (conidia) in fresh sterile distilled water. The final concentration of conidia was adjusted to $10 \times 10^{5} \mathrm{ml}^{-1}$. A suspension of conidia of Verticillium sp., the only fungus that could be cultured on agar was also prepared in the same manner using mycelia scraped from a 6-day old culture. Drops $(0.05 \mathrm{ml})$ of suspensions of conidia were applied separately on to the surface of three mature leaves of a healthy papaya tree. Six such trees were inoculated. To provide moist environment the leaves were covered with polyethylene bags soon after inoculation. The bags were removed after 48 hours. The leaves were observed both visually and microscopically for symptoms and confirmation of the causal organism.

\section{RESULTS AND DISCUSSION}

In 1992 we observed unusual black spotting in mature leaves of several papaya cultivars grown in certain areas around Kandy and the examination of diseased leaves revealed that the main causal agent was Asperisporium caricae. There were two other fungi found associated with the disease at different times of the year. These two were identified as Sclerospora sp. and Verticillium sp. The papaya cultivars were not known by name but all of them were common and popular local cultivars. Later in 1993 the disease rapidly spread to several areas within the Province and is now common in many other cultivars including Solo Hawaii. Artificial inoculation of conidia of $A$. caricae on to healthy leaves of papaya resulted in characteristic black spotting generally associated with the disease within 7-10 days. Verticillium sp. alone or together with conidia of $A$. caricae did not cause any infection. Similarly Sclerospora sp. failed to initiate infections.

The uppermost young leaves were completely free of infection. The disease was observed below the 10th or 12th leaf and the severity of disease increased progressively downwards with increasing leaf maturity. The fungus was restricted to the lower surface of the leaf. The diseased upper leaves showed numerous isolated, small (1-2 mm), circular to irregular, black, powdery, slighty 
(a)

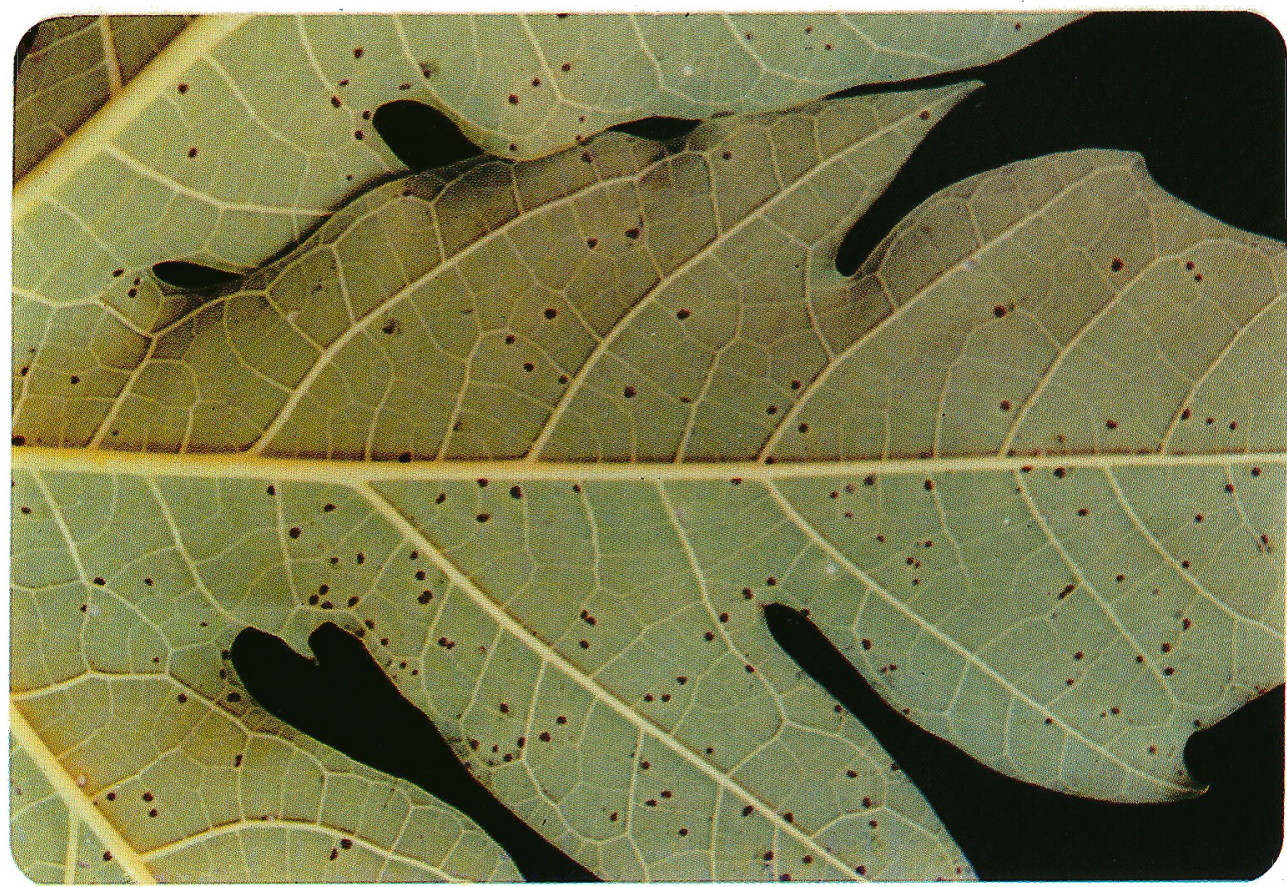

(b)

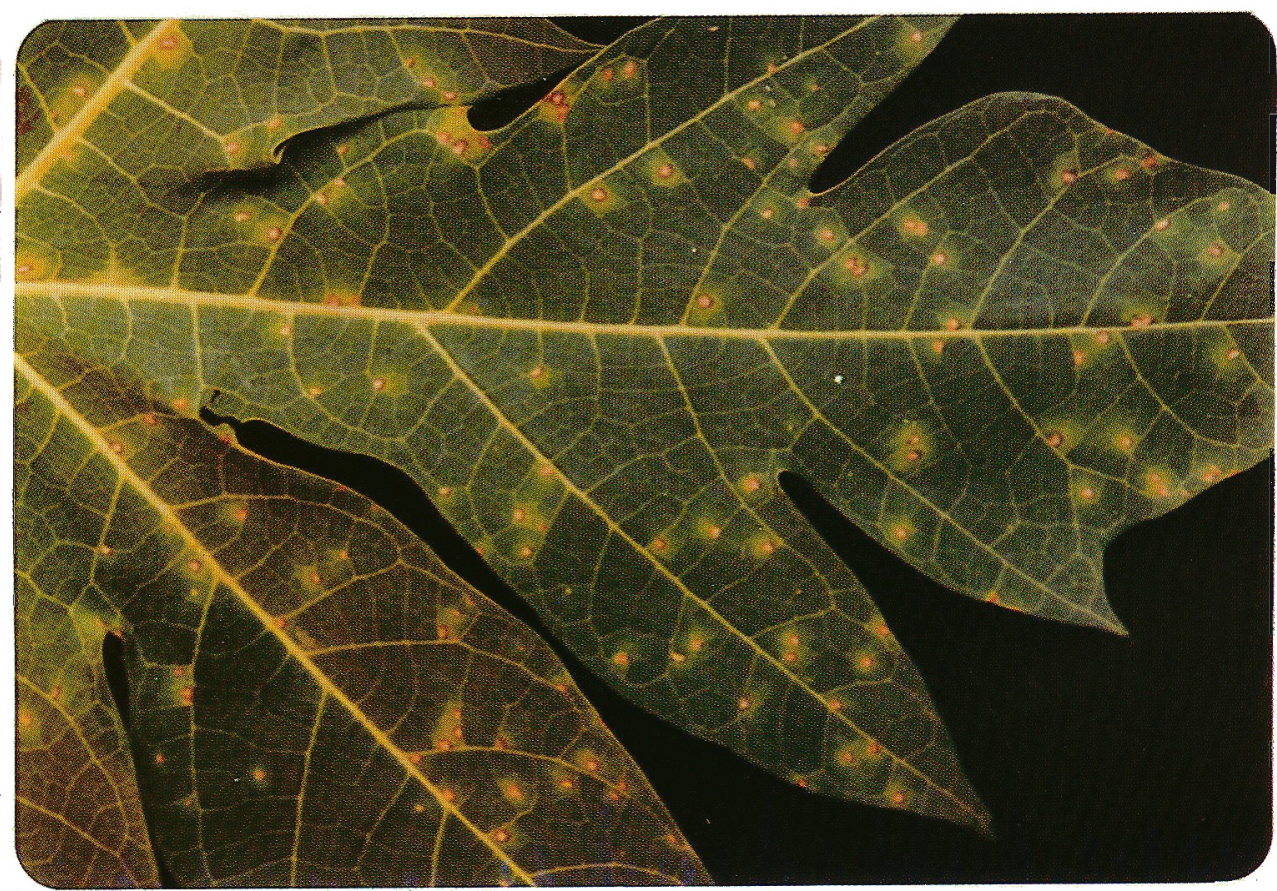


(c)

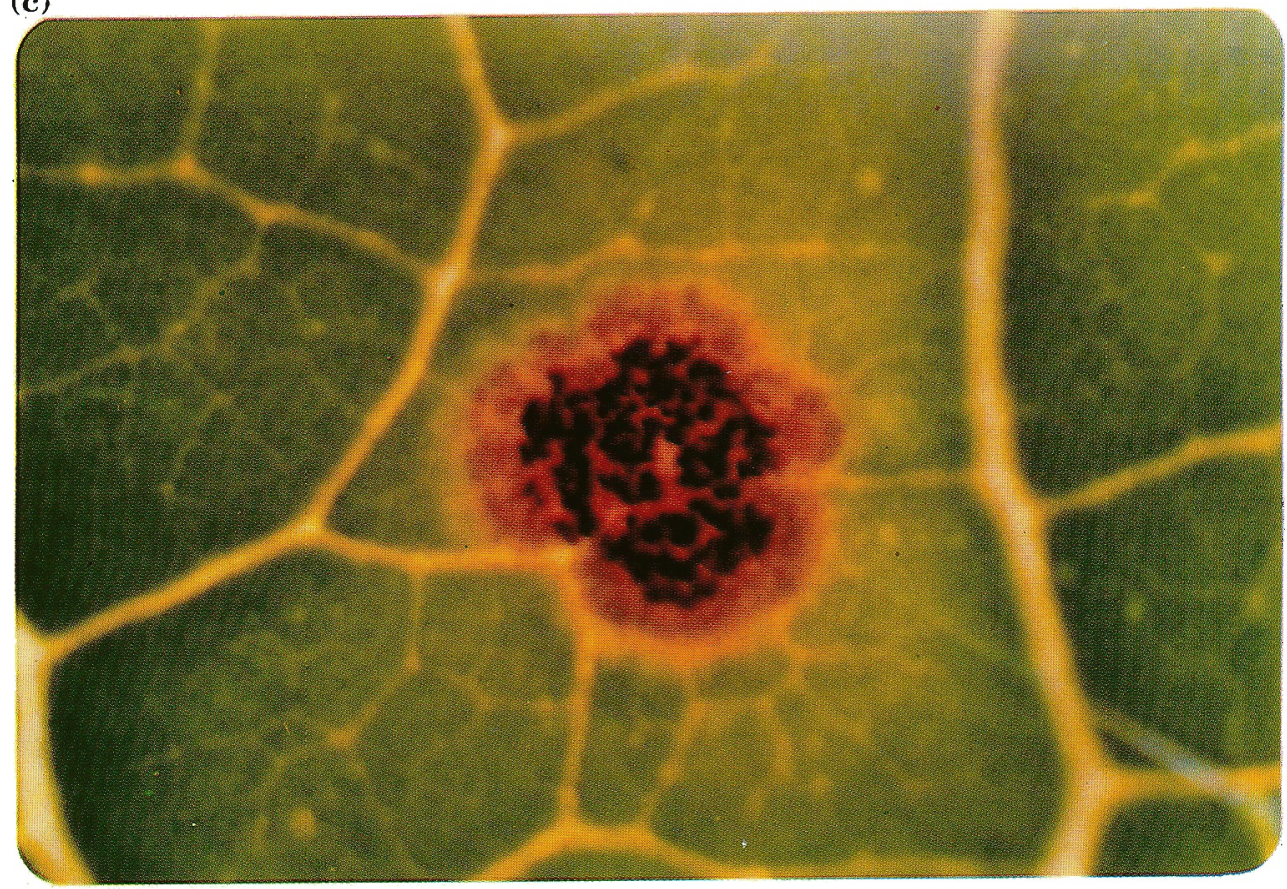

(d)

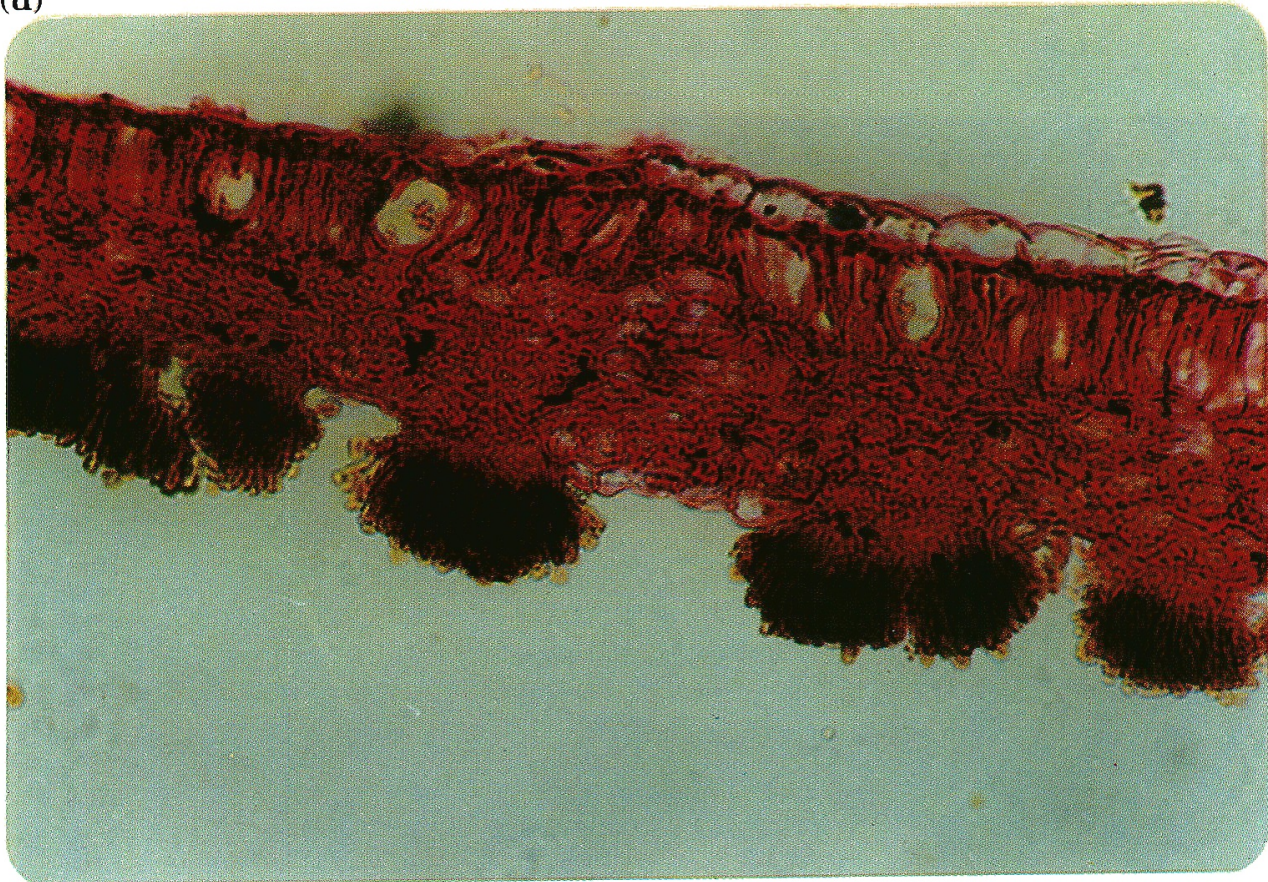

Figure 1: a) Lower surface; b) upper surface of a papaya leaf infected with $A$. caricae; c) an enlarged spot showing numerous, minute infection sites; d). transverse section of an infected papaya leaf showing clusters of closely packed conidiophores (X100). 
sunken spots (Fig. 1a). The upper surface of the diseased areas was necrotic (Fig. 1b). Severely.infected papaya trees occasionally blighted, the petioles and the leaves turned yellow and died. Individual spots were composed of 100-200 minute infected areas (Fig. 1c) each constituted aggregations of conidiophores (Fig. 1d). Thousands of conidia of $A$. caricae could be observed in these minute infected areas and the conidia were ellipsoidal, one-septate, pale brown or slightly black colour. The mycelium of the fungus is immersed and the conidiophores are closely packed together covering the surface of stromata and unbranched with prominent conidial scars at the apex. In more older leaves the infections were severe and the spots, which were isolated, scattered and rarely coalesced, appeared slightly larger (2-4 mm diameter). Attempts to isolate $A$. caricae or Sclerospore sp. on common mycological media viz. PDA, Cooks No. 2 Agar and Czapek Dox Agar, failed. Verticillium sp. was easily isolated from diseased spots in older leaves on these three media.

Sclerospora sp. and Verticillium sp., were observed in the majority of $A$. caricae spots in the more older leaves (Fig. 2a). Further their presence was particularly noticeable during the wet months. Sclerospora sp. produced cottony, greyish-white mildew growth and was confined only to the areas colonized by $A$. caricae and appeared growing adpressed to the mycelium of $A$. caricae (Fig. 2b). Verticillium sp. developed profuse, white, powdery mycelium on the Asperisporium spots. These two fungi had no contact with the host tissue. The exact role of these two fungi in the disease is not clear. However, their growth suggests the existence of some form of mycoparasitism.

(a)

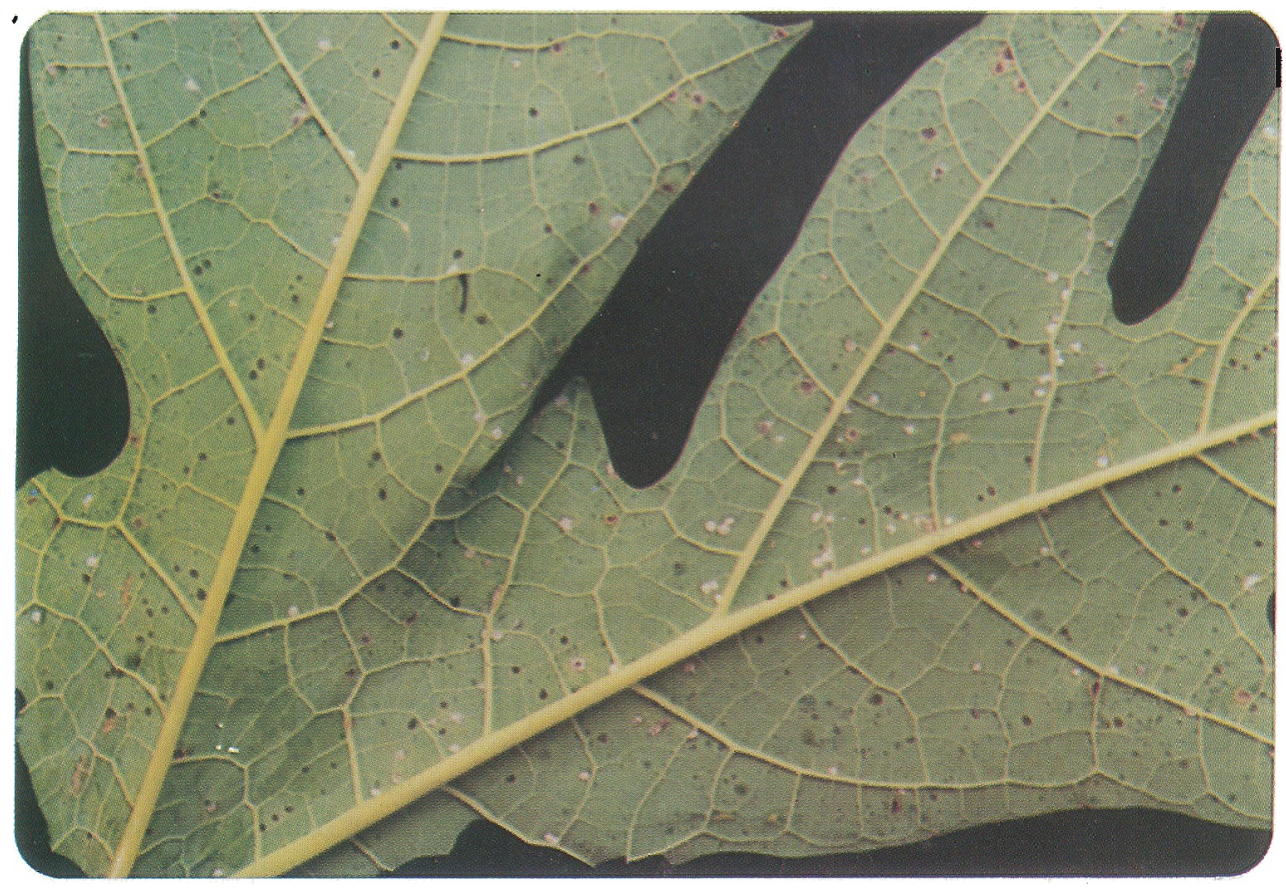


(b)

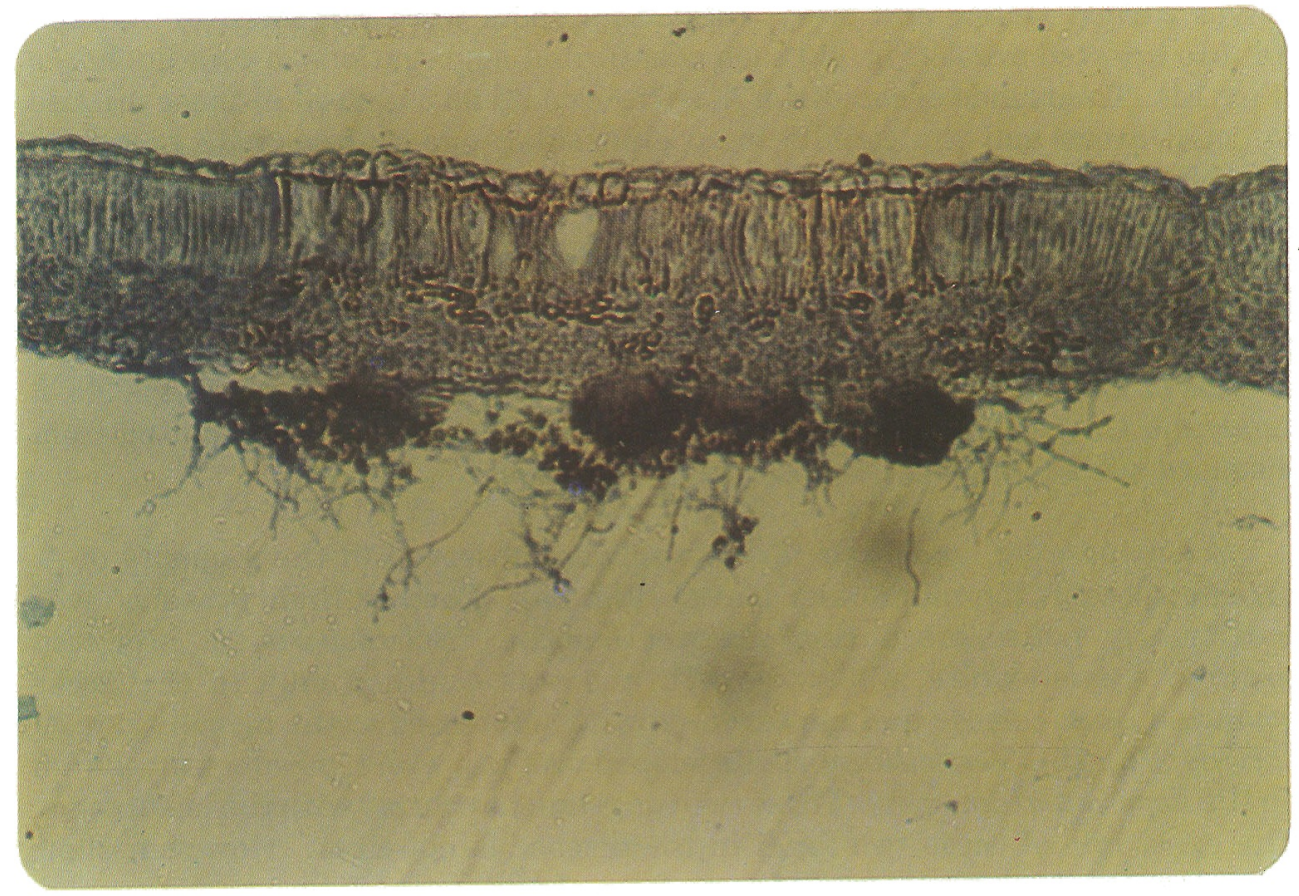

Figure 2: a) a portion; b) transverse section (x100) of an older leaf with A. caricae spots that have been subsequently colonized by Sclerospora sp. and Verticillium sp.

Occasionally the disease was also found in.papaya fruits, the symptoms were similar to those in the leaf. But the spots did not expand into progressive lesions during ripening. Sometimes very young fruits were also seen infected and this resulted in yellowing and premature fruit drop.

There was a marked correlation between the severity of Asperisporium spotting and the rainfall. Spotting increased considerably with increase in rainfall and during dry spells the disease declined markedly. During prolonged dry weather most infected trees recovered completely from the disease. However, even in the absence of rain, the fungi survived in fallen leaves and debris and caused infections with the commencement of rain. Incidence of the disease in Florida is also seasonal, most infections occurring in late winter and spring. ${ }^{1}$

The disease appears to be of considerable importance as the spotting greatly reduces the photosynthetic area and in severely infected plants the lower leaves turn brown, shrivel and take a twisted appearance, similar to a leaf blight. We have recently noticed sudden wilting followed by browning and complete death of the leaves and the petioles in the lower half of an infected papaya tree. 


\section{References}

1. Stevens J. (1939). Papaw diseases. Proceedings Florida Horticultural Society 52: 57-63.

2. Saldana M.I., Marquez M. \& Ruiz P. (1985). Identification de enfermedades fungosas del cultivo de la papaya (Carica papaya L. ). en el estado de Tabasco. Revista Mexicana de Fitopathologia 3: 14-17.

3. Ellis M.B. \& Holliday P. (1972). Asperisporium caricae. CMI Descriptions of Pathogenic Fungi and Bacteria, No. 347.

4. Chambers K.R. \& Rijkenfurg F.H.G. (1987). Culture of Asperisporium caricae, the papaya black spot organism. Phytophylactica 19: 113.

5. Snowden Anna L. (1990). A colour atlas of postharvest diseases and disorders of fruits and vegetables. Vol. 1: General introduction of fruits. Wolfe Scientific Ltd. London, $302 \mathrm{pp}$. 